JOURNAL OF

FUNCTION SPACES AND APPLICATIONS

Volume 3, Number 1 (2005), 73-89 (c) 2005, Scientific Horizon http://www.jfsa.net

\title{
On small Lebesgue spaces
}

\section{Claudia Capone and Alberto Fiorenza}

(Communicated by Pankaj Jain)

2000 Mathematics Subject Classification. 46E30, 46B10

Keywords and phrases. Grand Lebesgue spaces, small Lebesgue spaces, Banach Function Spaces, Orlicz spaces, fundamental function.

Abstract. We consider a generalized version of the small Lebesgue spaces, introduced in [5] as the associate spaces of the grand Lebesgue spaces. We find a simplified expression for the norm, prove relevant properties, compute the fundamental function and discuss the comparison with the Orlicz spaces.

\section{Introduction}

Let $\Omega \subset \mathbb{R}^{N}$ be a bounded domain and $f: \Omega \rightarrow \mathbb{R}^{N}, f=\left(f^{1}, \ldots, f^{N}\right)$ be a mapping of Sobolev class $W_{\text {loc }}^{1, N}\left(\Omega, \mathbb{R}^{N}\right)$. Let us denote by $D f(x)$ : $\mathbb{R}^{N} \rightarrow \mathbb{R}^{N}$ the differential and by $J(x, f)=\operatorname{det} D f(x)$ the Jacobian of $f$. After the elementary remark that by Hölder's inequality the Jacobian $J(x, f)$ is in $L_{l o c}^{1}(\Omega)$, the first fundamental result on the integrability of the Jacobian was due to Müller ([24]). He proved that if $f$ is an orientation preserving mapping, i.e. $J(x, f) \geq 0$ for a.e. $x \in \Omega$, then $J$ enjoys a greater degree of integrability, namely, it belongs locally to the Zygmund class $L \log L$. As a dual result, Iwaniec and Sbordone in [19] introduced the grand Lebesgue space $L^{N)}(\Omega)$, and proved that under the assumption 
$|D f| \in L^{N)}(\Omega)$ the Jacobian of $f$ is locally integrable. Such assumption, weaker than $|D f| \in L^{N}(\Omega)$, is shown to be minimal.

In many other papers the integrability properties of the Jacobian determinant has been studied, see e.g. [25], [19], [20], [21], [23], [22] and references therein. The grand Lebesgue spaces play an important role also in PDE theory, as shown by various papers ([3], [8], [9], [12], [15], [26], [27]).

The aim of this paper is to study the so-called small Lebesgue spaces $L^{(p}(\Omega)$, introduced in [5] and characterized as the associate space (see e.g. [2], [28]) of the grand Lebesgue spaces $L^{p)}(\Omega)$, defined setting

$$
\|f\|_{p)}=\sup _{0<\varepsilon<p-1}\left(\varepsilon f_{\Omega}|f|^{p-\varepsilon} d x\right)^{\frac{1}{p-\varepsilon}}
$$

where by $f_{\Omega}$ we denote $\frac{1}{|\Omega|} \int_{\Omega}$.

It turns out that the small Lebesgue spaces play a role in PDEs, too (see [10], [11]). The goal here is to give a simplified expression for the norm (see Theorem 3.3) and to give an easy proof of some new properties (see Corollaries 3.7, 3.8, 3.9, Proposition 4.2, Theorem 4.6, Propositions 5.4, $5.5)$. The main point here is that by Theorem 3.3 we provide an answer to a problem indirectly posed in the first paper [5] on small Lebesgue spaces. In order to explain such problem, we recall briefly how the definition of the norm has been introduced. First it has been considered the (auxiliary) space

$$
L^{\left(p^{\prime}\right.}(\Omega)=\left\{g \in \mathcal{M}_{o}:\||g|\|_{\left(p^{\prime}\right.}<+\infty\right\}
$$

where

$$
\|g\|_{\left(p^{\prime}\right.}=\inf _{g=\sum_{k=1}^{\infty} g_{k}}\left\{\sum_{k=1}^{\infty} \inf _{0<\varepsilon<p-1} \varepsilon^{-\frac{1}{p-\varepsilon}}\left(f_{\Omega}\left|g_{k}\right|^{(p-\varepsilon)^{\prime}} d x\right)^{\frac{1}{(p-\varepsilon)^{\prime}}}\right\},
$$

the functions $g_{k}, k \in \mathbf{N}$, being in $\mathcal{M}_{o}$, the set of all real, scalar-valued measurable functions, finite a.e. in $\Omega$. After this definition, the small Lebesgue spaces have been defined by

$$
L^{p)^{\prime}}(\Omega)=\left\{g \in \mathcal{M}_{o}:\|g\|_{p)^{\prime}}<+\infty\right\}
$$

where

$$
\|g\|_{p)^{\prime}}=\sup _{\substack{0 \leq \psi \leq|g| \\ \psi \in L^{\left(p^{\prime}\right.}(\Omega)}}\|\psi\|_{\left(p^{\prime}\right.}
$$


We remark that for this last space it has been proved the Fatou property, and therefore its coincidence with the associate space of $L^{p)}(\Omega)$, while this property has not been proved for the space $L^{\left(p^{\prime}\right.}(\Omega)$, leaving open the question whether the two spaces coincide or not, and, consequently, leaving open the possibility that the norm (1.2) can be written in the simplified way (1.1). The main result of this paper (see Theorem 3.3) is that the two spaces coincide and therefore $L^{\left(p^{\prime}\right.}(\Omega)$ is in fact the associate space of $L^{p)}(\Omega)$. As a consequence, we obtain some other new properties of the small Lebesgue spaces.

Let us mention here the recent paper [6], in which different, simple expressions (depending on the decreasing rearrangement) for the norm of the grand and small Lebesgue spaces have been found.

We will often deal ourselves with a generalization of the grand and small Lebesgue spaces, namely, the spaces $L^{p), \theta}(\Omega), \theta>0$, defined by

$$
\|f\|_{p), \theta}=\sup _{0<\varepsilon<p-1}\left(\varepsilon^{\theta} f_{\Omega}|f|^{p-\varepsilon} d x\right)^{\frac{1}{p-\varepsilon}}
$$

and the spaces $L^{\left(p^{\prime}, \theta\right.}(\Omega), \theta>0$, defined by

$$
\|g\|_{\left(p^{\prime}, \theta\right.}=\inf _{g=\sum_{k=1}^{\infty} g_{k}}\left\{\sum_{k=1}^{\infty} \inf _{0<\varepsilon<p-1} \varepsilon^{-\frac{\theta}{p-\varepsilon}}\left(f_{\Omega}\left|g_{k}\right|^{(p-\varepsilon)^{\prime}} d x\right)^{\frac{1}{(p-\varepsilon)^{\prime}}}\right\} .
$$

For $\theta=0$ it is $\|f\|_{p), 0}=\|f\|_{p}$ (this follows from the classical Hölder's inequality and Theorem 194, p. 143 of [16]), while $\|f\|_{\left(p^{\prime}, 0\right.}=\|f\|_{p^{\prime}}$ (this follows from the classical Hölder's and Minkowski's inequalities). For $\theta=1$ such spaces reduce obviously respectively to $L^{p)}(\Omega)$ and $L^{\left(p^{\prime}\right.}(\Omega)$.

In the paper we will assume that $\Omega$ is an open subset of $\mathbb{R}^{N}, N \geq 1$, $|\Omega|<+\infty ; p$ is any exponent in $] 1,+\infty\left[, p^{\prime}\right.$ is its Hölder conjugate exponent and, for all the results concerning the generalized spaces, $\theta$ is any positive number. All the proofs concerning the generalized spaces will be omitted if they are straightforward, otherwise, we will stress only the relevant differences.

The plan of the paper is the following. In Section 2 we will make a list of some basic results about Banach Function Space theory, which will be needed in the rest of the paper. In Section 3 we will establish the main result, and also that $L^{\left(p^{\prime}\right.}(\Omega)$ has absolutely continuous norm; moreover, we will show that the set of bounded functions is dense in $L^{\left(p^{\prime}\right.}(\Omega)$ and, finally, we will characterize the grand Lebesgue space $L^{p)}(\Omega)$ as dual space of $L^{\left(p^{\prime}\right.}(\Omega)$. In Section 4 we will exhibit the expression of the fundamental function of the generalized grand and small Lebesgue spaces. Finally, in 
Section 5, we will give embedding results between small Lebesgue spaces and Orlicz spaces.

\section{Preliminaries}

Let $\mathcal{M}_{o}^{+}$be the set of all measurable functions, whose values lie in $[0,+\infty]$, finite a.e. in $\Omega$.

Definition 2.1. A mapping $\rho: \mathcal{M}_{o}^{+} \rightarrow[0,+\infty]$ is called a Banach function norm if, for all $f, g, f_{n}(n=1,2,3, \ldots)$ in $\mathcal{M}_{o}^{+}$, for all constants $a \geq 0$, and for all measurable subset $E \subset \Omega$, the following properties hold:

$$
\begin{gathered}
\rho(f)=0 \Leftrightarrow f=0 \text { a.e. in } \Omega \\
\rho(a f)=a \rho(f) \\
\rho(f+g) \leq \rho(f)+\rho(g) \\
0 \leq g \leq f \text { a.e. in } \Omega \Rightarrow \rho(g) \leq \rho(f) \\
0 \leq f_{n} \uparrow f \text { a.e. in } \Omega \Rightarrow \rho\left(f_{n}\right) \uparrow \rho(f) \\
|E|<+\infty \Rightarrow \rho\left(\chi_{E}\right)<+\infty \\
|E|<+\infty \Rightarrow \int_{E} f d x \leq C_{E} \rho(f)
\end{gathered}
$$

for some constant $C_{E}, 0<C_{E}<\infty$, depending on $E$ and $\rho$, but independent of $f$.

Definition 2.2. If $\rho$ is a Banach function norm, the Banach space

$$
X=\left\{f \in \mathcal{M}_{o}: \rho(|f|)<+\infty\right\}
$$

is called a Banach Function Space.

For each $f \in X$ define

$$
\|f\|_{X}=\rho(|f|) .
$$

Examples of Banach Function Spaces are the familiar Lebesgue spaces and, more generally, the Orlicz spaces defined by

$$
\|f\|_{\Phi}=\inf \left\{\lambda>0: f_{\Omega} \Phi\left(\frac{|f|}{\lambda}\right) d x \leq 1\right\} .
$$

Definition 2.3. If $\rho$ is a function norm, its associate norm $\rho^{\prime}$ is defined on $\mathcal{M}_{o}^{+}$by

$$
\rho^{\prime}(g)=\sup \left\{\int_{\Omega} f g d x: f \in \mathcal{M}^{+}, \rho(f) \leq 1\right\} .
$$


Definition 2.4. Let $\rho$ be a function norm and let $X=X(\rho)$ be the Banach Function Space determined by $\rho$. Let $\rho^{\prime}$ be the associate norm of $\rho$. The Banach Function Space $X^{\prime}=X\left(\rho^{\prime}\right)$ determined by $\rho^{\prime}$ is called the associate space of $X$.

A standard example is that one of Orlicz spaces: the associate space of $L^{\Phi}(\Omega)$ is given by $L^{\tilde{\Phi}}(\Omega)$, where $\tilde{\Phi}$ denotes the complementary function of $\Phi$, defined by

$$
\tilde{\Phi}(t)=\max \{s t-\Phi(s): s \geq 0\} .
$$

In particular from the definition of $\|f\|_{X}$ it follows that the norm of a function $g$ in the associate space $X^{\prime}$ is given by

$$
\|g\|_{X^{\prime}}=\sup \left\{\int_{\Omega} f g d x: f \in \mathcal{M}^{+},\|f\|_{X} \leq 1\right\} .
$$

Theorem 2.5 ([2], Theorem 2.7, p. 10). Every Banach Function Space $X$ coincides with its second associate space $X^{\prime \prime}$.

Proposition 2.6 ([2], Proposition 2.10, p. 13). If $X$ and $Y$ are Banach Function Spaces and $X \subset Y$ (continuous embedding), then $Y^{\prime} \subset X^{\prime}$ (continuous embedding).

Definition 2.7. A function $f$ in a Banach Function Space $X$ is said to have absolutely continuous norm in $X$ if $\left\|f \chi_{E_{n}}\right\|_{X} \rightarrow 0$ for every sequence $\left\{E_{n}\right\}_{n=1}^{\infty}$ satisfying $E_{n} \downarrow \emptyset$ a.e. The set of all functions in $X$ of absolutely continuous norm is denoted by $X_{a}$. If $X=X_{a}$, then the space $X$ itself is said to have absolutely continuous norm.

Definition 2.8. Let $X$ be a Banach Function Space. The closure in $X$ of the set of bounded functions is denoted by $X_{b}$.

Theorem 2.9 ([2], Theorem 3.11, p. 18). Let $X$ be a Banach Function Space. Then $X_{a} \subseteq X_{b} \subseteq X$.

Corollary 2.10. If $X_{a}=X$, then $X_{b}=X$.

Theorem 2.11 ([2], Corollary 4.3, p. 23). The Banach space dual $X^{*}$ of a Banach Function Space $X$ is canonically isometrically isomorphic to the associate space $X^{\prime}$ if and only if $X$ has absolutely continuous norm.

\section{The equivalence between $L^{p)^{\prime}, \theta}(\Omega)$ and $L^{\left(p^{\prime}, \theta\right.}(\Omega)$}

For each $g \in \mathcal{M}_{o}$ let us set

$$
\|g\|_{p)^{\prime}, \theta}=\sup _{\substack{0 \leq \Psi \leq|g| \\ \Psi \in L\left(p^{\prime}, \theta(\Omega)\right.}}\|\Psi\|_{\left(p^{\prime}, \theta\right.}
$$


In [5] it is proved the following result, which we state here for the generalized case.

Theorem 3.1. The space defined by

$$
L^{p)^{\prime}, \theta}(\Omega)=\left\{g \in \mathcal{M}_{o}:\||g|\|_{p)^{\prime}, \theta}<+\infty\right\}
$$

is a Banach Function Space, and coincides with the associate space of $L^{p), \theta}(\Omega)$.

By using the same arguments as in [11], the following result holds.

Theorem 3.2. Let $\left(f_{m}\right)$ be a monotone nondecreasing sequence (i.e. $\left.f_{m} \leq f_{m+1}, m \in \mathbf{N}\right)$ such that $M=\sup _{m}\left\|f_{m}\right\|_{\left(p^{\prime}, \theta\right.}<+\infty$.

Then the function $f=\sup _{m} f_{m}$ is such that

$$
\begin{gathered}
f \in L^{\left(p^{\prime}, \theta\right.}(\Omega) \\
f_{m} \nearrow f \text { a.e. } \\
f_{m} \rightarrow f \text { in } L^{\left(p^{\prime}, \theta\right.}(\Omega)
\end{gathered}
$$

Now our aim is to prove the following

Theorem 3.3. The space $L^{\left(p^{\prime}, \theta\right.}(\Omega)$ is a Banach Function Space and

$$
L^{p)^{\prime}, \theta}(\Omega) \equiv L^{\left(p^{\prime}, \theta\right.}(\Omega)
$$

and therefore

$$
\|g\|_{p)^{\prime}, \theta}=\inf _{g=\sum_{k=1}^{\infty} g_{k}}\left\{\sum_{k=1}^{\infty} \inf _{0<\varepsilon<p-1} \varepsilon^{-\frac{\theta}{p-\varepsilon}}\left(f_{\Omega}\left|g_{k}\right|^{(p-\varepsilon)^{\prime}} d x\right)^{\frac{1}{(p-\varepsilon)^{\prime}}}\right\} .
$$

Proof. Let us observe that since $\|g\|_{\left(p^{\prime}, \theta\right.}$ is an order preserving norm then

$$
\|\Psi\|_{\left(p^{\prime}, \theta\right.} \leq\|f\|_{\left(p^{\prime}, \theta\right.} \quad \forall \Psi \in L^{\left(p^{\prime}, \theta\right.}(\Omega), \quad \Psi \leq|f|
$$

hence

$$
\|f\|_{p)^{\prime}, \theta} \leq\|f\|_{\left(p^{\prime}, \theta\right.} .
$$


Let us prove the other inequality. To this aim let $f$ be such that $\|f\|_{p)^{\prime}, \theta}<\infty$, otherwise there is nothing to prove. Set

$$
f_{n}=\min \{|f|, n\} .
$$

The sequence $\left(f_{n}\right)$ verifies the hypothesis of Theorem 3.2 , so for $|f|=$ $\sup _{n} f_{n}$ we have $|f| \in L^{\left(p^{\prime}, \theta\right.}(\Omega)$, and replacing $\Psi$ by $|f|$ in the definition of $\|f\|_{p)^{\prime}, \theta}$ we obtain

$$
\|f\|_{p)^{\prime}, \theta} \geq\|f \mid\|_{\left(p^{\prime}, \theta\right.}=\|f\|_{\left(p^{\prime}, \theta\right.} .
$$

The proof is over.

As a consequence of Theorem 3.1 and Theorem 3.3, we get

Corollary 3.4. The following Hölder's inequality holds

$$
f_{\Omega} f g d x \leq\|f\|_{p), \theta}\|g\|_{\left(p^{\prime}, \theta\right.}
$$

and $\|\cdot\|_{\left(p^{\prime}, \theta\right.}$ is the smallest norm for which (3.11) holds.

Remark 3.5. As a matter of fact only the second part of the statement follows from Theorem 3.1 and Theorem 3.3; the proof of inequality (3.11) is a straightforward modification of the proof of Theorem 2.5 in [5].

We will need in the following next lemma, proved in [5] in the case $\theta=1$.

Lemma 3.6. Let $F_{n} \subset \Omega, n \in \mathbf{N}$, be such that $\chi_{F_{n}} \downarrow 0$ a.e. in $\Omega$ and let $g$ be any function in $L^{\left(p^{\prime}, \theta\right.}(\Omega)$. Then

$$
\left\|g \chi_{F_{n}}\right\|_{\left(p^{\prime}, \theta\right.} \rightarrow 0 .
$$

Corollary 3.7. The Banach Function Space $L^{\left(p^{\prime}, \theta\right.}(\Omega)$ has absolutely continuous norm.

Proof. By Theorem 3.3 the space $L^{\left(p^{\prime}, \theta\right.}(\Omega)$ is a Banach Function Space, and by Lemma 3.6 such space has absolutely continuous norm.

Corollary 3.8. The set of bounded functions is dense in $L^{\left(p^{\prime}, \theta\right.}(\Omega)$.

Proof. By Corollary 3.7 the space $L^{\left(p^{\prime}, \theta\right.}(\Omega)$ has absolutely continuous norm. Since by Corollary 2.10 the space $L^{\left(p^{\prime}, \theta\right.}(\Omega)$ coincides with the closure of the bounded functions, the result is proved. 
Corollary 3.9. The dual of $L^{\left(p^{\prime}, \theta\right.}(\Omega)$ is canonically isometrically isomorphic to the associate space of $L^{\left(p^{\prime}, \theta\right.}(\Omega)$ and the following relation holds

$$
\left(L^{\left(p^{\prime}, \theta\right.}(\Omega)\right)^{*}=\left(L^{\left(p^{\prime}, \theta\right.}(\Omega)\right)^{\prime}=L^{p), \theta}(\Omega)
$$

Proof. The first equality follows directly from Corollary 3.7 and Theorem 2.11. The second is consequence of Theorem 3.3 and Theorem 2.5.

\section{The fundamental function}

Let $f \in \mathcal{M}_{o}$. The function

$$
\mu_{f}(\lambda)=|\{x \in \Omega:|f(x)|>\lambda\}| \quad \forall \lambda \geq 0
$$

is called the distribution function of $f$. The decreasing rearrangement of $f$, $f^{*}$, is defined on $[0,|\Omega|]$ by

$$
f^{*}(t)=\inf \left\{\lambda>0: \mu_{f}(\lambda) \leq t\right\}
$$

where here we use the convention $\inf \emptyset=+\infty$. Two functions having the same distribution function are called equimisurable. Let us recall that a function norm $\rho$ is said to be rearrangement-invariant (briefly, "r.i.") if $\rho(f)=\rho(g)$ for every couple of equimisurable functions. The Banach Function Space arising from a r.i. function norm is called a r.i. space. For each $t \in] 0,|\Omega|]$, let $E_{t} \subseteq \Omega$ be a set of measure $t$. The fundamental function of $X, \varphi_{X}(t)$, is defined by

$$
\varphi_{X}(t)=\rho\left(\chi_{E_{t}}\right)
$$

The following result holds ([2], Corollary 4.4, p. 60, and Theorem 5.2, p. 66)

Theorem 4.1. Let $X$ be a r.i. space and let $X^{\prime}$ its associate space. Then $X^{\prime}$ is r.i. and

$$
\left.\left.\varphi_{X}(t) \varphi_{X^{\prime}}(t)=t \quad \forall \quad t \in\right] 0,|\Omega|\right] .
$$

Our first observation here is that after Theorem 3.3 we are able to show, by using a very simple argument, that the norm of $L^{\left(p^{\prime}, \theta\right.}(\Omega)$ is r.i. We remark that recently a different proof has been obtained in [6].

Proposition 4.2. $L^{\left(p^{\prime}, \theta\right.}(\Omega)$ is a r.i. space. 
Proof. Since $L^{p}(\Omega)$ is a r.i. space, then $L^{p), \theta}(\Omega)$ is also a r.i. space. Moreover, by Theorem 4.1, also $L^{p)^{\prime}, \theta}(\Omega)$ is a r.i. space. Finally, by Theorem 3.3 , we get the assertion.

The following is a classical result ([2], Lemma 8.17, p. 276), but we recall it for completeness.

Lemma 4.3. Let $L^{B}(\Omega)$ be an Orlicz space. Then the fundamental function is

$$
\left.\left.\varphi(t)=\frac{1}{B^{-1}\left(\frac{1}{t}\right)}, \quad \forall t \in\right] 0,|\Omega|\right] .
$$

As a consequence, we get

Corollary 4.4. The fundamental function of $\frac{L^{p}}{(\log L)^{\theta}}(\Omega)$ is equivalent to

$$
\varphi(t) \approx t^{\frac{1}{p}}\left[\log \left(\frac{1}{t}\right)\right]^{-\frac{\theta}{p}}
$$

as $t \rightarrow 0+$.

The following result shows that $L^{p), \theta}(\Omega)$ and $\frac{L^{p}}{(\log L)^{\theta}}(\Omega)$ have the same fundamental function, so that, in some sense, such spaces are "very close" each other.

Theorem 4.5. Let $\varphi$ be the fundamental function of the space $L^{p), \theta}(\Omega)$. Then we have

$$
\varphi(t) \approx t^{\frac{1}{p}}\left[\log \left(\frac{1}{t}\right)\right]^{-\frac{\theta}{p}}
$$

as $t \rightarrow 0+$.

Proof. The proof is essentially analogous to the case $\theta=1$, shown in [5]. We give here only a few details concerning some differences with the simpler case $\theta=1$.

The fundamental function of $L^{p), \theta}(\Omega)$ reads as

$$
\varphi(t)=\sup _{0<\epsilon<p-1}\left(\epsilon^{\theta} t\right)^{\frac{1}{p-\epsilon}} .
$$

Defining

$$
F_{\theta}(\epsilon)=\left(\epsilon^{\theta} t\right)^{\frac{1}{p-\epsilon}}
$$


we obtain

$$
F_{\theta}^{\prime}(0+)=\left\{\begin{array}{ll}
+\infty & \text { if } \theta<p \\
t^{\frac{1}{p}} & \text { if } \theta=p \\
0 & \text { if } \theta>p
\end{array} \quad F_{\theta}^{\prime}(p-1)<0\right.
$$

In either case one can still verify that $F_{\theta}^{\prime}$ is, up to a positive factor, a decreasing function, positive in some $\left(0, \epsilon_{t}\right)$ and negative in $\left(\epsilon_{t}, p-1\right)$. Therefore there exists a unique point of maximum $\epsilon_{t}$ of $F_{\theta}$ and the proof can proceed exactly as in the case $\theta=1$.

We conclude this section giving the expression of the fundamental function for the small Lebesgue spaces. The result is direct consequence of Theorem 4.5 and Theorem 4.1.

Theorem 4.6. Let $\varphi$ be the fundamental function of the space $L^{\left(p^{\prime}, \theta\right.}(\Omega)$. Then we have

$$
\varphi(t) \approx t^{\frac{1}{p^{t}}}\left[\log \left(\frac{1}{t}\right)\right]^{\frac{\theta}{p}}
$$

as $t \rightarrow 0+$.

Remark 4.7. The Theorem 4.6 shows that in some sense $L^{\left(p^{\prime}, \theta\right.}(\Omega)$ is "very close" to $L^{p^{\prime}}(\log L)^{\frac{\theta}{p-1}}(\Omega)$.

\section{Orlicz embeddings for small Lebesgue spaces}

In [18] the authors find sharp results on the inclusions between grand Lebesgue spaces (case $\theta=1$ ) and Orlicz spaces. We will state such results, in the general case $\theta>0$, splitting them into the following propositions.

Proposition 5.1. Let $B(t)$ be a Young function. If

$$
B(t) \geq c \frac{t^{p}}{\log ^{\theta} t}
$$

for large $t$, then the continuous embedding holds:

$$
L^{B}(\Omega) \subset L^{p), \theta}(\Omega) .
$$

Proof. From the embedding theorems between Orlicz spaces (see e.g. [1], Theorem 8.12, p. 234), we have $L^{B}(\Omega) \subset \frac{L^{p}}{(\log L)^{\theta}}(\Omega)$. Since $\frac{L^{p}}{(\log L)^{\theta}}(\Omega) \subset$ $L^{p), \theta}(\Omega)$ (this is a simple generalization of the argument for $\theta=1$ in [18]), we get $L^{B}(\Omega) \subset L^{p), \theta}(\Omega)$. 
Remark 5.2. The condition (5.1), in the case $\theta=1$, is shown to be optimal in [18], proving in fact the finer inclusion $L^{B}(\Omega) \subset V L^{p}(\Omega)$, where $V L^{p}(\Omega)$ stands for the closure of $L^{\infty}(\Omega)$ in $L^{p)}(\Omega)$. We notice that it is possible to prove the optimality also in the following, simple, way.

For each $t \in] 0,|\Omega|]$, let $E_{t} \subset \Omega$ be a set of measure $t$. Since $L^{B}(\Omega) \subset$ $L^{p), \theta}(\Omega)$, then, in particular,

$$
\left.\left.\left\|\chi_{E_{t}}\right\|_{p), \theta} \leq c\left\|\chi_{E_{t}}\right\|_{B} \quad \forall t \in\right] 0,|\Omega|\right]
$$

hence, by Lemma 4.3 and Corollary 4.4,

$$
\left.\left.t^{\frac{1}{p}}\left[\log \left(\frac{1}{t}\right)\right]^{-\frac{\theta}{p}} \leq c \frac{1}{B^{-1}\left(\frac{1}{t}\right)} \quad \forall t \in\right] 0,|\Omega|\right]
$$

which implies (5.1).

Proposition 5.3. Let $B(t)$ be a Young function such that

$$
t \rightarrow \frac{B(t) \log ^{\theta} t}{t^{p}}
$$

is decreasing for large $t$.

If

$$
\int_{1}^{+\infty} \frac{B(t)}{t^{p+1} \log ^{1-\theta}(e+t)} d t<+\infty
$$

then the continuous embedding holds:

$$
L^{p), \theta}(\Omega) \subset L^{B}(\Omega) .
$$

Proof. The following argument follows the ideas of [18]. Suppose that $B$ satisfies (5.2). Set

$$
F(t)=\frac{B(t) \log ^{\theta} t}{t^{p}}
$$

Consider an arbitrary $u \in L^{p), \theta}(\Omega),\|u\|_{p), \theta}=1$ and set

$$
\Omega_{k}=\left\{x \in \Omega: e^{e^{k}} \leq|u(x)|<e^{e^{k+1}}\right\} \quad k=0,1,2, \ldots
$$

so that

$$
e \leq|u(x)|^{e^{-k}}<e^{e} \quad \forall x \in \Omega_{k} .
$$


Since $F(t)$ is decreasing for $t \geq e^{e^{\nu}}$ for some $\nu$ large, we have

$$
\begin{aligned}
\int_{|u| \geq e^{e^{\nu}}} B(|u|) d x & =\sum_{k=\nu}^{\infty} \int_{\Omega_{k}} B(|u|) d x \\
& =\sum_{k=\nu}^{\infty} \int_{\Omega_{k}} \frac{u^{p} F(u)}{\log ^{\theta} u} d x \\
& \leq \sum_{k=\nu}^{\infty} \int_{\Omega_{k}} \frac{u^{p} F\left(e^{e^{k}}\right)}{e^{k \theta}} d x \\
& \leq e^{e} \sum_{k=\nu}^{\infty} F\left(e^{e^{k}}\right) e^{-k \theta} \int_{\Omega_{k}} u^{p-e^{-k}} d x \\
& \leq e^{e}|\Omega| \sum_{k=\nu}^{\infty}\|u\|_{p), \theta}^{p-e^{-k}} F\left(e^{e^{k}}\right) \\
& =e^{e}|\Omega| \sum_{k=\nu}^{\infty} F\left(e^{e^{k}}\right) \\
& \leq e^{e}|\Omega| \int_{0}^{\infty} F\left(e^{e^{t}}\right) d t \\
& =e^{e}|\Omega| \int_{e}^{\infty} \frac{F(t)}{t \log t} d t \\
& \leq e^{e}|\Omega| \int_{1}^{\infty} \frac{B(t)}{t^{p+1} \log { }^{1-\theta} t} d t<\infty
\end{aligned}
$$

therefore $u \in L^{B}(\Omega)$.

From the previous results, by a standard duality argument (using Proposition 2.6, Theorem 3.1 and Theorem 3.3), we obtain the following two propositions.

Proposition 5.4. Let $B(t)$ be a Young function. If

$$
B(t) \leq c t^{p^{\prime}} \log \frac{\theta}{p-1} t
$$

for large $t$, then the continuous embedding holds:

$$
L^{\left(p^{\prime}, \theta\right.}(\Omega) \subset L^{B}(\Omega) .
$$


Proposition 5.5. Let $B(t)$ be a Young function and $\widetilde{B}(t)$ be its complementary function. If

$$
\int_{1}^{+\infty} \frac{\widetilde{B}(t)}{t^{p+1} \log ^{1-\theta}(e+t)} d t<+\infty
$$

then the continuous embedding holds:

$$
L^{B}(\Omega) \subset L^{\left(p^{\prime}, \theta\right.}(\Omega) .
$$

As a consequence of the previous statements we get the following result, used in [4]:

Proposition 5.6. The following continuous embeddings hold:

$$
\bigcup_{\beta>1} L^{p}(\log L)^{\frac{\beta \theta}{p^{\prime}-1}}(\Omega) \subset L^{(p, \theta}(\Omega) \subset L^{p}(\log L)^{\frac{\theta}{p^{\prime}-1}}(\Omega) .
$$

Moreover, both inclusions in (5.5) are proper.

Proof. Inclusions (5.5) follow directly from Proposition 5.4 and Proposition 5.5. We now show that such inclusions are proper. Regarding the first inclusion, it is sufficient to observe that for instance

$$
\bigcup_{\beta>1} L^{p}(\log L)^{\frac{\beta \theta}{p^{\prime}-1}}(\Omega) \varsubsetneqq L^{p}(\log L)^{\frac{\theta}{p^{\prime}-1}}(\log \log L)^{\frac{2}{p^{\prime}-1}}(\Omega) \varsubsetneqq L^{(p, \theta}(\Omega)
$$

where the second inclusion is due to Proposition 5.5.

We now show that the inclusion

$$
L^{(p, \theta}(\Omega) \subset L^{p}(\log L)^{\frac{\theta}{p^{\prime}-1}}(\Omega)
$$

is proper. We provide two motivations for such statement.

First, observe that the two spaces are different, because their associate spaces are different: in fact in [15] it is declared that in $L^{p), \theta}(\Omega)$ the space $C_{0}^{\infty}(\Omega)$ is not dense, while this applies for the associate space of $L^{p}(\log L)^{\frac{\theta}{p^{\prime}-1}}(\Omega)$.

A second, direct, proof consists in showing a function $f \in L^{p), \theta}(\Omega)$, $f \notin \frac{L^{p}}{(\log L)^{\theta}}(\Omega)$, and observing that the conclusion follows by duality (Proposition 2.6 and Theorem 2.5).

Consider $\Omega=] 0,1 / 2[\subset \mathbb{R}$ and set

$$
f(x)=\frac{1}{x^{1 / p} \log ^{(1-\theta) / p}\left(\frac{1}{x}\right)}
$$


It is $f \notin \frac{L^{p}}{(\log L)^{\theta}}(\Omega)$ because

$$
\frac{f^{p}}{\log ^{\theta} f} \approx \frac{1}{x \log \left(\frac{1}{x}\right)} \notin L^{1}(\Omega)
$$

On the other hand, by $[6]$

$$
\|f\|_{p), \theta} \approx \sup _{0<t<1}(1-\log t)^{-\frac{\theta}{p}}\left(\int_{t}^{1}\left[f^{*}(s)\right]^{p} d s\right)^{\frac{1}{p}}
$$

and therefore $f \in L^{p), \theta}(\Omega)$ because

$$
\left(\int_{t}^{1}\left[f^{*}(s)\right]^{p} d s\right)^{\frac{1}{p}}=\left(\frac{1}{\theta}\right)^{\frac{1}{p}} \log ^{\frac{\theta}{p}}\left(\frac{1}{t}\right) .
$$

Remark 5.7. The spaces $L^{p), \theta}$ and $L^{\left(p^{\prime}, \theta\right.}$ considered in this paper, which represent a generalization of the original grand and small Lebesgue spaces, have been used in the theory of PDEs (see [15]) and are going to be used in extrapolation theory (see [4]). Looking at the results of this paper and at the literature on this subject, it is natural to consider even a more general class of spaces, whose definition is the following.

Let $p>1$ and $\delta:] 0, p-1[\rightarrow] 0, \infty[$ be an increasing function such that $\delta(0+)=0$. Then the functionals

$$
\|f\|_{p), \delta}=\sup _{0<\varepsilon<p-1}\left(\delta(\varepsilon) f_{\Omega}|f|^{p-\varepsilon} d x\right)^{\frac{1}{p-\varepsilon}}
$$

and

$$
\|g\|_{\left(p^{\prime}, \delta\right.}=\inf _{g=\sum_{k=1}^{\infty} g_{k}}\left\{\sum_{k=1}^{\infty} \inf _{0<\varepsilon<p-1}\left(\frac{1}{\delta(\varepsilon)}\right)^{\frac{1}{p-\varepsilon}}\left(f_{\Omega}\left|g_{k}\right|^{(p-\varepsilon)^{\prime}} d x\right)^{\frac{1}{(p-\varepsilon)}}\right\}
$$

define respectively the new spaces $L^{p), \delta}$ and $L^{\left(p^{\prime}, \delta\right.}$.

We believe that all the results already proved for $L^{p), \theta}$ and $L^{\left(p^{\prime}, \theta\right.}$, in this paper as well as in the previous papers on this subject (see e.g. [5, 6, 13, 10, $11,14,18,19])$, can be extended without too much effort in this new setting, and that such a refiniment can give a good contribution for the applications actually known (see e.g. $[3,4,8,9,10,11,12,14,15,17,19,26,27]$ ). On the other hand, while it can be more or less straightforward to make a generalization of single results, requiring eventually to the function $\delta$ to satisfy some additional hypotheses, it remains open the problem to find the 
most general assumption on $\delta$ such that at the same time all the relevant results of the theory still hold, and some relevant questions can be still solved, for instance:

1. $L^{p), \delta}$ and $L^{\left(p^{\prime}, \delta\right.}$ are Banach Function Spaces.

2. The associate space of $L^{p), \delta}$ is $L^{\left(p^{\prime}, \delta\right.}$.

3. The dual space of $L^{\left(p^{\prime}, \delta\right.}$ is isometrically isomorphic to $L^{p), \delta}$.

4. Bounded functions are dense in $L^{\left(p^{\prime}, \delta\right.}$ while are not in $L^{p), \delta}$.

5. The norms of $L^{p), \delta}$ and $L^{\left(p^{\prime}, \delta\right.}$ can be expressed explicitely in terms of rearrangements.

6. The fundamental functions of $L^{p), \delta}$ and $L^{\left(p^{\prime}, \delta\right.}$ can be computed explicitely.

7. The comparison of $L^{p), \delta}$ and $L^{\left(p^{\prime}, \delta\right.}$ with Orlicz Spaces.

8. The Sobolev-type theorem for functions whose gradient is in $L^{N), \delta}$ and $L^{(N, \delta}$.

\section{References}

[1] R.A. Adams, Sobolev spaces, Academic Press, 1975.

[2] C. Bennett and R. Sharpley, Interpolation of Operators, Academic Press, 1988.

[3] L. Boccardo, Quelques problemes de Dirichlet avec donnees dans de grands espaces de Sobolev, C. R. Acad. Sci. Paris Sér I Math., 325 (1997), 1269-1272.

[4] C. Capone, A. Fiorenza and M. Krbec, On extrapolation blowups in the $L_{p}$ scale, to appear.

[5] A. Fiorenza, Duality and reflexivity in grand Lebesgue spaces, Collect. Math., 51 (2) (2000), 131-148.

[6] A. Fiorenza and G. E. Karadzhov Grand and small Lebesgue spaces and their analogs, preprint Istituto per le Applicazioni del Calcolo "Mauro Picone" del C.N.R., sezione di Napoli, 272 (3) (2003), Z. Anal. Anwendungen, to appear.

[7] A. Fiorenza and M. Krbec On the domain and range of the maximal operator, Nagoya Math. J., 158 (2000) , 43-61.

[8] A. Fiorenza, A. Mercaldo and J.M. Rakotoson, Regularity and Comparison Results in Grand Sobolev Spaces for Parabolic Equations with Measure Data, Applied Mathematics Letters, 14 (2001), 979-981.

[9] A. Fiorenza, A. Mercaldo and J.M. Rakotoson, Regularity and uniqueness results in Grand Sobolev Spaces for parabolic equations with measure data, Discrete and Continuous Dynamical Systems, 8 (4) (2002), 893-906. 
[10] A. Fiorenza and J.M. Rakotoson, Petit espaces de Lebesgue et quelques applications, C. R. Acad. Sci. Paris Sér I Math., 334 (2002), 23-26.

[11] A. Fiorenza and J.M. Rakotoson, New properties of Small Lebesgue Spaces and their Applications, Math. Ann., 326 (2003), 543-561.

[12] A. Fiorenza and C. Sbordone, Existence and uniqueness results for solutions of nonlinear equations with right hand side in $L^{1}$, Studia Math., 127 (3) (1998), 223-231.

[13] N. Fusco, P.L. Lions and C. Sbordone, Sobolev imbedding theorems in borderline cases, Proc. Amer. Math. Soc., 124 (2) (1996), 561-565.

[14] L. Greco, A remark on the equality $\operatorname{det} D f=\operatorname{Det} D F$, Differential Integral Equations, 6 (1993), 1089-1100.

[15] L. Greco, T. Iwaniec and C. Sbordone Inverting the p-harmonic operator, Manuscripta Math., 92, (1997), 249-258.

[16] G.H Hardy, J.E. Littlewood and G. Pólya, Inequalities, Cambridge University Press, 1952.

[17] P. Koskela and X. Zhong, Minimal assumptions for the integrability of the Jacobian, Ricerche di Matematica, 51 (2) (2002), 297-311.

[18] T. Iwaniec, P. Koskela and J. Onninen, Mappings of finite distorsion: Monotonicity and continuity, Invent. Math., 144 (2001), 507-531.

[19] T. Iwaniec and C. Sbordone, On the integrability of the Jacobian under minimal hypothesis, Arch. Rat. Mech. Anal., 119 (1992), 129-143.

[20] T. Iwaniec and C. Sbordone, Quasiharmonic Fields, Ann. I. H. Poincaré - AN 18 (5) (2001), 519-572.

[21] T. Iwaniec and A. Verde, A study of Jacobians in Hardy-Orlicz spaces, Proc. Roy. Soc. Edinburgh Sect. A, 129 (3) (1999), 539-570.

[22] J. Kauhanen, P. Koskela and J. Maly, Mappings of finite distortion: Condition N., Michigan Math. J., 49 (2001), 169-181.

[23] G. Moscariello, On the integrability of the Jacobian in Orlicz spaces, Math. Japonica, 40 (2) (1994), 323-329.

[24] S. Müller, A surprising higher integrability property of mappings with positive determinant, Bull. Amer. Math. Soc., 21 (1989), 245-248.

[25] S. Müller, Higher integrability of determinants and weak convergence in $L^{1}$, J. Reine Angew. Math., 412 (1990), 20-34.

[26] C. Sbordone, Nonlinear Elliptic Equations with Right Hand Side in Nonstandard Spaces, Atti Sem. Mat. Fis. Univ. Modena, Suppl. to, 46 (1988), 361-368.

[27] C. Sbordone, Grand Sobolev spaces and their applications to variational problems, Le Matematiche, 51 (2) (1996), 335-347.

[28] A.C. Zaanen, Integration, North-Holland, 1967. 
C.N.R. Istituto per le Applicazioni del Calcolo "Mauro Picone" sezione di Napoli, via P.Castellino 111

80131 Napoli

Italia

(E-mail : capone@na.iac.cnr.it)

Dipartimento di Costruzioni e Metodi Matematici in Architettura Università di Napoli

via Monteoliveto 3

80134 Napoli

Italia

and

C.N.R. Istituto per le Applicazioni del Calcolo "Mauro Picone" sezione di Napoli

via P.Castellino 111

80131 Napoli

Italia (E-mail : fiorenza@unina.it) 


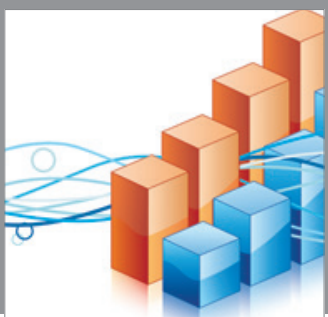

Advances in

Operations Research

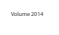

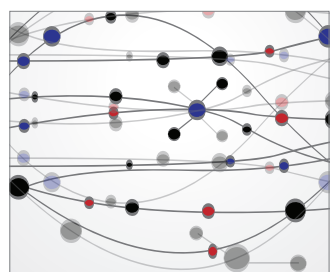

\section{The Scientific} World Journal
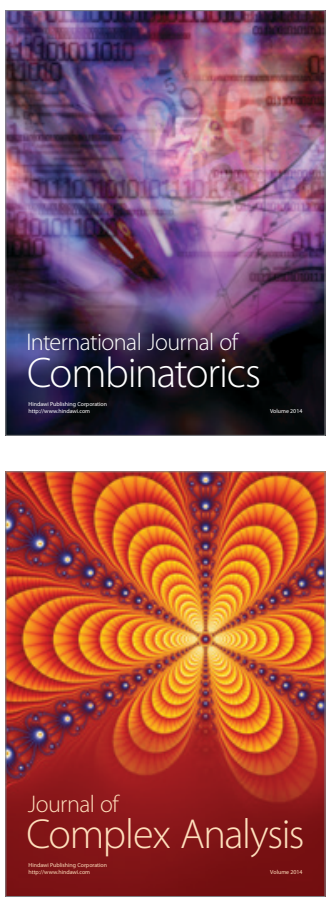

International Journal of

Mathematics and

Mathematical

Sciences
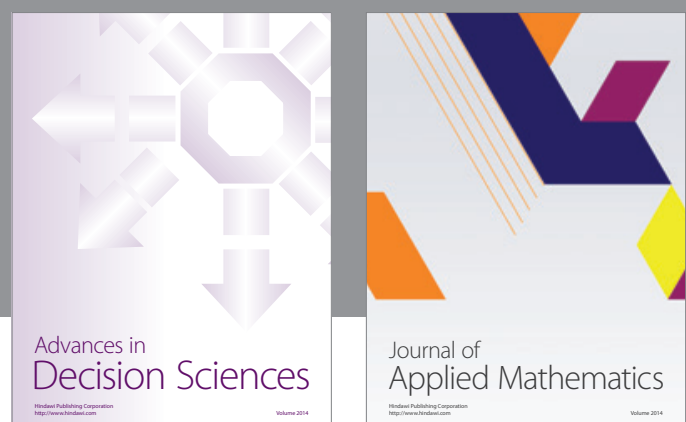

Journal of

Applied Mathematics
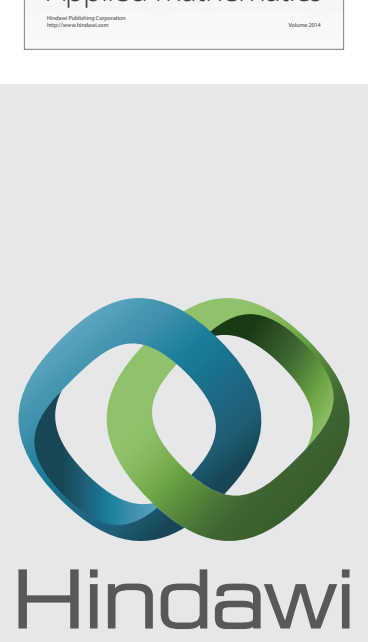

Submit your manuscripts at http://www.hindawi.com
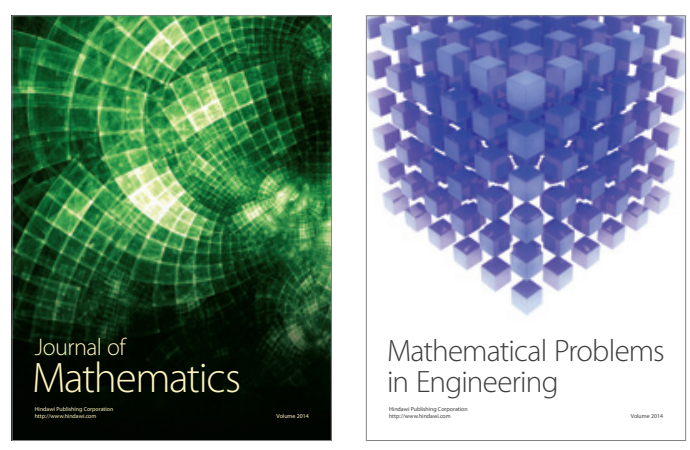

Mathematical Problems in Engineering
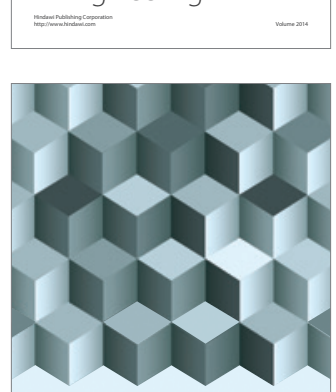

Journal of

Function Spaces
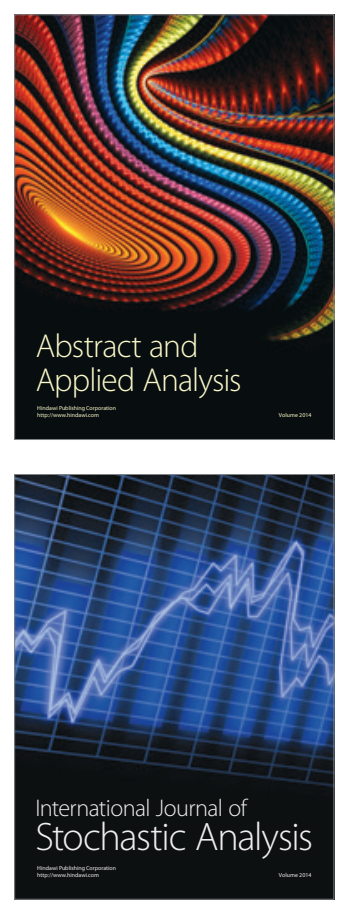

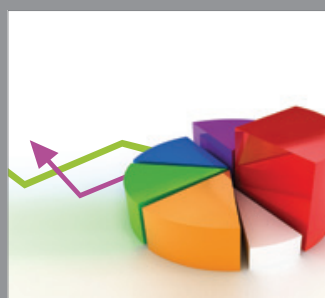

ournal of

Probability and Statistics

Promensencen
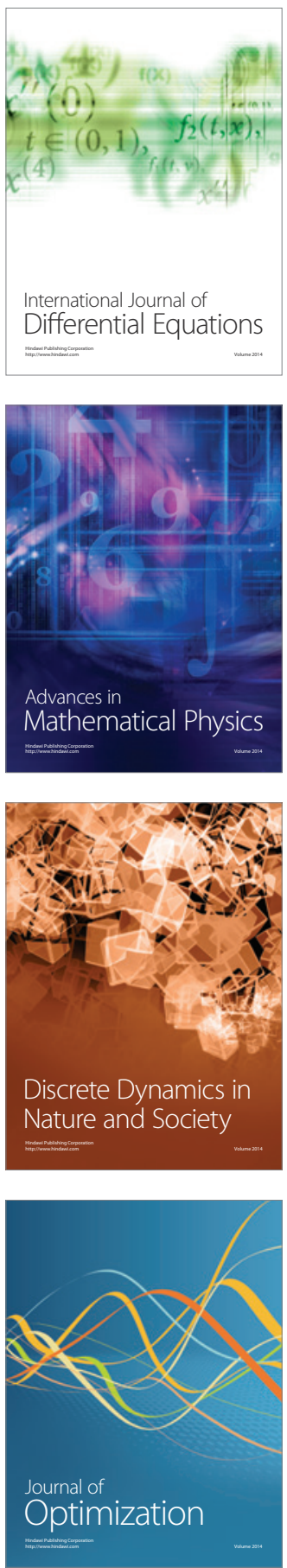